\title{
Some results on $\chi$-single valued neutrosophic subgroups
}

\author{
M. Shazib Hameed ${ }^{1}$, Zaheer Ahmad ${ }^{2}$, Salman Mukhtar ${ }^{3}$, Asad Ullah ${ }^{4}$ \\ ${ }^{1,2}$ Department of Mathematics, Khwaja Fareed University of Engineering \& Information Technology, Rahim Yar Khan, \\ Punjab 64200, Pakistan \\ ${ }^{3}$ Department of Mathematics, The Islamia University of Bahawalpur, Punjab 63100, Pakistan \\ ${ }^{4}$ Department of Mathematics, The Islamia University of Bahawalpur, Rahim Yar Khan Campus, Punjab 64200, Pakistan
}

\begin{tabular}{l}
\hline Article Info \\
\hline Article history: \\
Received Mar 16, 2021 \\
Revised Jul 9, 2021 \\
Accepted Jul 13, 2021 \\
\hline
\end{tabular}

Keywords:

Neutrosophic $\chi$-single valued neutrosophic set

$\chi$-single valued neutrosophic subgroups

\section{Corresponding Author:}

Muhammad Shazib Hameed

Department of Mathematics

Khwaja Fareed University of Engineering \& Information Technology

Rahim Yar Khan, Punjab 64200, Pakistan

Email: shazib.hameed@kfueit.edu.pk

\begin{abstract}
In this study, we develop a novel structure $\chi$-single valued neutrosophic set, which is a generalization of the intuitionistic set, inconsistent intuitionistic fuzzy set, Pythagorean fuzzy set, spherical fuzzy set, paraconsistent set, etc. Fuzzy subgroups play a vital role in vagueness structure, it differ from regular subgroups in that it is impossible to determine which group elements belong and which do not. In this paper, we investigate the concept of a $\chi$-single valued neutrosophic set and $\chi$-single valued neutrosophic subgroups. We explore the idea of $\chi$-single valued neutrosophic set on fuzzy subgroups and several characterizations related to $\chi$-single valued neutrosophic subgroups are suggested.
\end{abstract}

This is an open access article under the CC BY-SA license.

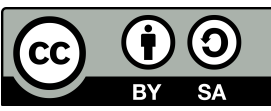

\section{INTRODUCTION}

In general, the drawbacks of previously developed methods and models are mitigated by the newly defined fuzzy algebraic structure. Because of the limitations of routine mathematics, it cannot always be used. Certain daily systems have vague and missing information. Methodologies were seen as an alternative to dealing with these issues and preventing flaws, such as certainty, rough set, and a fuzzy set hypothesis. Unfortunately, each of these alternative mathematics has flaws and drawbacks, such as the majority of terms like true, beautiful, and popular, which are not readily identifiable or even ambiguous. As a result, the rules for such terms can differ from one person to the next.

Zadeh [1] has begun an analysis of the possibility based on the participation feature assigning a registration grade in $[0,1]$ in order to deal with such unclear and uncertain information. Atanassov [2] suggested that intuitionistic fuzzy sets could be used as a fuzzy set extension in lieu of the concepts of enrolment and non-participation. Molodtsov [3] coined the term soft set to describe a computational model for dealing with uncertainties. Because of its applications in a variety of lively topics, the possibility of soft set has gained a new destination for scientists. Crisp sets have two independent generalizations: fuzzy sets and soft sets. In the soft set hypothesis, Ali et al. [4] suggested several new operations. They discussed extended and restricted union and intersection. Yager [5]-[7] first proposed the Pythagorean fuzzy set. Few Pythagorean fuzzy data intrusions interventions have been developed and implemented by Peng et al. [8]. Peng et al. looked at Pythagorean fuzzy soft sets and how they were implemented in [9]. The variety of models were investigated in [10]-[14]. 
Arockiarani and Jency [15] studied the basic characteristics of fuzzy neutrosophic sets and also introduced the fuzzy neutrosophic topological spaces. They also explored the properties of the respective developed spaces [16]. This concept is extended for the groups and various algebraic structures as given in [17]-[29]. The paper is arranged as follows: In Section 2, we give some basic concepts related to fuzzy single-valued neutrosophic sets $(S V N S s)$. In Sections 3 and 4, we introduce the notion of $\chi$-single valued neutrosophic sets $(\chi-S V N S s)$ and $\chi$-single valued neutrosophic subgroups respectively, and also proposed several characterizations on $\chi$-single valued neutrosophic subgroups.

\section{PRELIMINARIES}

Definition 2..1. [15] A $S V N S L$ on the universe set $S$ is defined as: $L=\left\{\left\langle u, \alpha_{L}(u), \beta_{L}(u), \gamma_{L}(u)\right\rangle, u \in\right.$ $S\}$ where $\alpha, \beta, \gamma: S \rightarrow[0,1]$ and $0 \leq \alpha_{L}(u)+\beta_{L}(u)+\gamma_{L}(u) \leq 3$.

Definition 2..2. [15] Let $S$ be a non empty set, and $L=\left\{\left\langle u, \alpha_{L}(u), \beta_{L}(u), \gamma_{L}(u)\right\rangle\right\}, M=\left\{\left\langle u, \alpha_{M}(u)\right.\right.$, $\left.\left.\beta_{M}(u), \gamma_{M}(u)\right\rangle\right\}$ be $S V N S s$, then proceeding properties must satisfy:

(i) $L \subseteq M, \forall u$ if $\alpha_{L}(u) \leq \alpha_{M}(u), \beta_{L}(u) \leq \beta_{M}(u), \gamma_{L}(u) \geq \gamma_{M}(u)$.

(ii) $L \cup M=\left\langle u, \bigvee\left(\alpha_{L}(u), \alpha_{M}(u)\right), \bigvee\left(\beta_{L}(u), \beta_{M}(u)\right), \bigwedge\left(\gamma_{L}(u), \gamma_{M}(u)\right)\right\rangle$

(iii) $L \cap M=\left\langle u, \bigwedge\left(\alpha_{L}(u), \alpha_{M}(u)\right), \bigwedge\left(\beta_{L}(u), \beta_{M}(u)\right), \bigvee\left(\gamma_{L}(u), \gamma_{M}(u)\right)\right\rangle$.

(iv) $L \backslash M(u)=\left\langle u, \bigwedge\left(\alpha_{L}(u), \gamma_{M}(u)\right), \bigwedge\left(\beta_{L}(u), 1-\beta_{M}(u)\right), \bigvee\left(\gamma_{L}(u), \alpha_{M}(u)\right)\right\rangle$.

Definition 2..3. [15] A SVNS L is called null or empty $S V N S$ over the universe $S$ if $\alpha_{L}(u)=0, \beta_{L}(u)=0, \gamma_{L}(u)=1, \forall u \in S$. It is indicated with $O_{N}$.

Definition 2..4. [15] A SVNS of $L$ is an absolute $S V N S$ over the universe of $S$, if $\alpha_{L}(u)=1, \beta_{L}(u)=1, \gamma_{L}(u)=0, \forall u \in S$. It is indicated with $1_{N}$.

Definition 2..5. [15] $L^{c}$ is the complement of $S V N S L$ which is defined as $L^{c}=\left\langle u, \alpha_{L^{c}}(u), \beta_{L^{c}}(u), \gamma_{L^{c}}(u)\right\rangle$ where $\alpha_{L^{c}}(u)=\gamma_{L}(u), \beta_{L^{c}}(u)=1-\beta_{L}(u), \gamma_{L^{c}}(u)=\alpha_{L}(u)$. It is also possible to describe the complement of the SVNS L as $L^{c}=1_{N}-L$.

\section{3. $\chi$-SINGLE VALUED NEUTROSOPHIC SETS}

Definition 3..1. Consider $L=\left\{\left\langle u, \alpha_{L}(u), \beta_{L}(u), \gamma_{L}(u)\right\rangle, u \in S\right\}$, then $\chi-S V N S L^{\chi}$ on the discourse universe $S$ is defined as $L^{\chi}=\left\{\left\langle\alpha_{L}^{\chi}(u)=\bigwedge\left\{\alpha_{L}(u), \chi\right\}, \beta_{L}^{\chi}(u)=\bigwedge\left\{\beta_{L}(u), \chi\right\}, \gamma_{L}^{\chi}(u)=\bigvee\left\{\gamma_{L}(u), \chi\right\}\right\rangle, u \in\right.$ $S\}$ and $0 \leq \alpha_{L}^{\chi}(u)+\beta_{L}^{\chi}+\gamma_{L}^{\chi} \leq 3$, where $\chi \in[0,1]$, where $\alpha, \beta, \gamma: L \rightarrow[0,1]$.

Definition 3..2. Let $S$ be a non empty set, and $L^{\chi}=\left\langle\alpha_{L}^{\chi}(u)=\bigwedge\left\{\alpha_{L}(u), \chi\right\}, \beta_{L}^{\chi}(u)=\bigwedge\left\{\beta_{L}(u), \chi\right\}, \gamma_{L}^{\chi}(u)=\right.$ $\left.\bigvee\left\{\gamma_{L}(u), \chi\right\}\right\rangle, M^{\chi}=\left\langle\alpha_{M}^{\chi}(u)=\bigwedge\left\{\alpha_{M}(u), \chi\right\}, \beta_{M}^{\chi}(u)=\bigwedge\left\{\beta_{M}(u), \chi\right\}, \gamma_{M}^{\chi}(u)=\bigvee\left\{\gamma_{M}(u), \chi\right\}\right\rangle$, then following conditions must hold

(i) $L^{\chi} \subseteq M^{\chi}, \forall u$ if $\alpha_{L}^{\chi}(u) \leq \alpha_{M}^{\chi}(u), \beta_{L}^{\chi}(u) \leq \beta_{M}^{\chi}(u), \gamma_{L}^{\chi}(u) \geq \gamma_{M}^{\chi}(u)$.

(ii) $L^{\chi} \cup M^{\chi}=\left\langle u, \bigvee\left(\alpha_{L}^{\chi}(u), \alpha_{M}^{\chi}(u)\right), \bigvee\left(\beta_{L}^{\chi}(u), \beta_{M}^{\chi}(u)\right), \bigwedge\left(\gamma_{L}^{\chi}(u), \gamma_{M}^{\chi}(u)\right)\right\rangle$

(iii) $L^{\chi} \cap M^{\chi}=\left\langle u, \bigwedge\left(\alpha_{L}^{\chi}(u), \alpha_{M}^{\chi}(u)\right), \bigwedge\left(\beta_{L}^{\chi}(u), \beta_{M}^{\chi}(u)\right), \bigvee\left(\gamma_{L}^{\chi}(u), \gamma_{M}^{\chi}(u)\right)\right\rangle$

(iv) $L^{\chi} \backslash M^{\chi}(u)=\left\langle u, \bigwedge\left(\alpha_{L}^{\chi}(u), \gamma_{M}^{\chi}(u)\right), \bigwedge\left(\beta_{L}^{\chi}(u), 1-\beta_{M}^{\chi}(u)\right), \bigvee\left(\gamma_{L}^{\chi}(u), \alpha_{M}^{\chi}(u)\right)\right\rangle$.

Definition 3..3. A $\chi-S V N S L^{\chi}$ is called null or empty $\chi-S V N S$ over the universe $S$ if $\alpha_{L}^{\chi}(u)=0, \beta_{L}^{\chi}(u)=$ $0, \gamma_{L}^{\chi}(u)=1, \forall u \in S$. It is indicated with $O_{N}$.

Definition 3..4. A $\chi-S V N S$ of $L^{\chi}$ is an absolute $\chi-S V N S$ over the universe of $S$ if $\alpha_{L}^{\chi}(u)=1, \beta_{L}^{\chi}(u)=1, \gamma_{L}^{\chi}(u)=0, \forall u \in S$. It is indicated with $1_{N}$.

Definition 3..5. $L^{c^{\chi}}$ is the complement of $\chi-S V N S L^{\chi}$ which is defined as $L^{c^{\chi}}=\left\langle u, \alpha_{L^{c}}^{\chi}(u), \beta_{L^{c}}^{\chi}(u), \gamma_{L^{c}}^{\chi}(u)\right\rangle$ where $\alpha_{L^{c}}^{\chi}(u)=\gamma_{L}^{\chi}(u), \beta_{L^{c}}^{\chi}(u)=1-\beta_{L}^{\chi}(u), \gamma_{L^{c}}^{\chi}(u)=\alpha_{L}^{\chi}(u)$. Complement of the $\chi-S V N S L^{\chi}$ is $L^{c^{\chi}}=$ $1_{N}-L^{\chi}$.

Definition 3..6. Let $S$ and $T$ be two non-empty set, Define a function $g: S \rightarrow T$. (i) If $M^{\chi}=\left\{\left\langle v, \alpha_{M}^{\chi}(v), \beta_{M}^{\chi}\right.\right.$ $\left.(v), \gamma_{M}^{\chi}(v)\right\rangle: v$ in $\left.T\right\}$ be a $\chi-S V N S$ in $T$, then $g^{-1}\left(M^{\chi}\right)$ is a pre-image of $M^{\chi}$ under $g$ be a $\chi-S V N S$ in $S$ as described $g^{-1}\left(M^{\chi}\right)=\left\{\left\langle u, g^{-1}\left(\alpha_{M}^{\chi}(u)\right), g^{-1}\left(\beta_{M}^{\chi}(u)\right), g^{-1}\left(\gamma_{M}^{\chi}(u)\right)\right\rangle: u\right.$ in $\left.S\right\}$ where $g^{-1}\left(\alpha_{M}^{\chi}(u)\right)=$ $\alpha_{M}^{\chi}(g(u))$. (ii) If $L^{\chi}=\left\{\left\langle u, \alpha_{L}^{\chi}(u), \beta_{L}^{\chi}(u), \gamma_{L}^{\chi}(u)\right\rangle: u\right.$ in $\left.S\right\}$ be a $\chi-S V N S$ in $S$ then under $g$ the image of 
$L^{\chi}$ is denoted by $g\left(L^{\chi}\right)$, is the $\chi-S V N S$ in $T$ as described $g\left(L^{\chi}\right)=\left\{\left\langle v, g\left(\alpha_{L}^{\chi}(v)\right), g\left(\beta_{L}^{\chi}(v)\right), g_{\sim}\left(\gamma_{L}^{\chi}(v)\right)\right\rangle\right.$ : $v$ in $T\}$

$$
\begin{gathered}
g\left(\alpha_{L}^{\chi}(v)\right)=\left\{\begin{array}{cc}
\sup _{u \in g^{-1}(v)} \alpha_{L}^{\chi}(u) \quad, \text { if } g^{-1}(v) \neq 0_{N} \\
0 \quad, \text { otherwise }
\end{array} g_{L}^{\chi}(v)\right)= \begin{cases}\sup _{u \in g^{-1}(v)} \beta_{L}^{\chi}(u) & , \text { if } g^{-1}(v) \neq 0_{N} \\
0 & \text { otherwise }\end{cases} \\
g_{\sim}\left(\gamma_{L}^{\chi}(v)\right)= \begin{cases}\inf _{u \in g^{-1}(v)} \gamma_{L}^{\chi}(u) & , \text { if } g^{-1}(v) \neq 0_{N} \\
1 & \text { otherwise }\end{cases}
\end{gathered}
$$

and $g_{\sim}\left(\gamma_{L}^{\chi}(v)\right)=\left(1-g\left(1-\gamma_{L}^{\chi}\right)\right)_{v}$.

Definition 3..7. Consider $L^{\chi}$ is a $\chi-S V N S$ in group $(S,$.$) . Then L^{\chi}$ is said to be $\chi$-single valued neutrosophic group (in short, $\chi-S V N G$ ) in $S$ if it fulfill these two conditions: (i) $\alpha_{L}^{\chi}(u v) \geq \alpha_{L}^{\chi}(u) \wedge \alpha_{L}^{\chi}(v), \quad \beta_{L}^{\chi}(u v) \geq$ $\beta_{L}^{\chi}(u) \wedge \beta_{L}^{\chi}(v)$ and $\gamma_{L}^{\chi}(u v) \leq \gamma_{L}^{\chi}(u) \wedge \gamma_{L}^{\chi}(v)(i i) \alpha_{L}^{\chi}\left(u^{-1}\right) \geq \alpha_{L}^{\chi}(u), \beta_{L}^{\chi}\left(u^{-1}\right) \geq \beta_{L}^{\chi}(u), \gamma_{L}^{\chi}\left(u^{-1}\right) \leq \gamma_{L}^{\chi}(u)$.

Definition 3..8. let $L^{\chi}$ and $M^{\chi}$ be two $\chi-S V N S s$ in $S$ where $(S,$.$) be a groupoid, Then the \chi$-single valued neutrosophic product of $L^{\chi}$ and $M^{\chi}, L^{\chi} \circ M^{\chi}$ is defined as follows: for any $u \in S$,

$$
\begin{aligned}
& \alpha_{L \circ M}^{\chi}(u)= \begin{cases}\bigvee_{v w=u}\left[\alpha_{L}^{\chi}(v) \wedge \alpha_{M}^{\chi}(w)\right] & , \text { for each }(v, w) \in S \times S \text { with } v w=u, \\
0 & , \text { otherwise }\end{cases} \\
& \beta_{L \circ M}^{\chi}(u)= \begin{cases}\bigvee_{v w=u}\left[\beta_{L}^{\chi}(v) \wedge \beta_{M}^{\chi}(w)\right] & , \text { for each }(v, w) \in S \times S \text { with } v w=u, \\
0 & , \text { otherwise }\end{cases} \\
& \gamma_{L \circ M}^{\chi}(u)= \begin{cases}\bigwedge_{v w=u}\left[\gamma_{L}^{\chi}(v) \wedge \gamma_{M}^{\chi}(w)\right] & , \text { for each }(v, w) \in S \times S \text { with } v w=u, \\
1 & , \text { otherwise. }\end{cases}
\end{aligned}
$$

Definition 3..9. Consider $L^{\chi} \in \chi-S V N S(G)$ and $G$ be a groupoid. Then $L^{\chi}$ is called: (1) $\chi$-single valued neutrosophic left ideal ( $\chi-S V N L I)$ of $G$ if for some $u, v \in G, L^{\chi}(u v) \geq L^{\chi}(v)$. (i.e.,) $\alpha_{L}^{\chi}(u v) \geq$ $\alpha_{L}^{\chi}(v), \beta_{L}^{\chi}(u v) \geq \beta_{L}^{\chi}(v)$, and $\gamma_{L}^{\chi}(u v) \leq \gamma_{L}(v)(2) \chi$-single valued neutrosophic right ideal $(\chi-S V N R I)$ of $G$ iffor some $u, v \in G, L^{\chi}(u v) \geq L^{\chi}(u)$. (i.e.,) $\alpha_{L}^{\chi}(u v) \geq \alpha_{L}^{\chi}(u), \beta_{L}(u v) \geq \beta_{L}^{\chi}(u)$, and $\gamma_{L}^{\chi}(u v) \leq \gamma_{L}^{\chi}(u)$ (3) $\chi$-single valued neutrosophic ideal $(\chi-S V N I)$ of $G$ if it is $\chi$-SVNLI as well as $\chi-S V N R I$ Clearly, $L^{\chi}$ is a $\chi-S V N I$ of $G \Leftrightarrow$ for any $u, v \in G, \alpha_{L}^{\chi}(u v) \geq \alpha_{L}^{\chi}(u) \vee \alpha_{L}^{\chi}(v), \beta_{L}^{\chi}(u v) \geq \beta_{L}^{\chi}(u) \vee \beta_{L}^{\chi}(v)$, and $\gamma_{L}^{\chi}(u v) \leq$ $\gamma_{L}^{\chi}(u) \wedge \gamma_{L}^{\chi}(v)$. Furthermore, a $\chi-S V N I$ (respectively $\chi-S V N L I, \chi-S V N R I$ ) is a single valued $\chi-$ neutrosophic subgroupoid $\chi-S V N S G P$ of $G$. Remember for every $\chi-S V N S G P L^{\chi}$ of $G$ we get $\alpha_{L}^{\chi}\left(u^{n}\right) \geq$ $\alpha_{L}^{\chi}(u), \beta_{L}^{\chi}\left(u^{n}\right) \geq \beta_{L}^{\chi}(u)$, and $\gamma_{L}^{\chi}\left(u^{n}\right) \leq \gamma_{L}^{\chi}(u)$ for every $u \in G$, while $u^{n}$ is any composite of $u^{\prime} s$. The collection of all $\chi-S V N S G P$ s with $G$ will be denoted as $\chi-S V N S G P(G)$.

Definition 3..10. Let $(G,$.$) be a groupoid and assume O_{N} \neq L^{\chi} \in \chi-S V N S(G)$ Then $L^{\chi}$ is called a $\chi$-single valued neutrosophic subgroupoid in $G(\chi-S V N S G P$ in $G)$ if $L^{\chi} \circ L^{\chi} \subset L^{\chi}$.

Definition 3..11. Let $(G,$.$) be a groupoid and consider L^{\chi} \in \chi-S V N S(G)$. Then $L^{\chi}$ is said to be $\chi$ $S V N S G P$ in $G$, iffor every $u, v \in G, \alpha_{L}^{\chi}(u v) \geq \alpha_{L}^{\chi}(u) \vee \alpha_{L}^{\chi}(v), \beta_{L}^{\chi}(u v) \geq \beta_{L}^{\chi}(u) \vee \beta_{L}^{\chi}(v)$, and $\gamma_{L}^{\chi}(u v) \leq$ $\gamma_{L}^{\chi}(u) \wedge \gamma_{L}^{\chi}(v)$. Clearly $0_{N}$ and $1_{N}$ are both $\chi-S V N S G P$ s of $G$.

Definition 3..12. Let $L^{\chi} \in \chi-S V N S(G)$. If for any $\alpha \in P(G), \exists a t_{0} \in \alpha$ such that $L^{\chi}\left(t_{0}\right)=\bigcup_{t \in \alpha}\left(L^{\chi}(t)\right)$ $t_{0} \in \alpha$ such that $L^{\chi}\left(t_{0}\right)=\bigcup_{t \in \alpha}\left(L^{\chi}(t)\right)$.i.e., $\alpha_{L}^{\chi}\left(t_{0}\right)=\bigvee_{t \in \alpha}\left(\alpha_{L}^{\chi}(t)\right), \quad \beta_{L}^{\chi}\left(t_{0}\right)=\bigvee_{t \in \alpha}\left(\beta_{L}^{\chi}(t)\right), \gamma_{L}^{\chi}\left(t_{0}\right)=$ $\bigwedge_{t \in \alpha}\left(\gamma_{L}^{\chi}(t)\right)$, where $P(G)$ denote the power set of $G$. then we called $L^{\chi}$ have a sup-property.

Definition 3..13. Let $L^{\chi}$ be a $\chi-S V N S$ in $S$ and let $\varpi, \delta, v \in \beta$ with $\varpi+\delta+v \leq 3$. Then the set $S_{L \chi}^{(\varpi, \delta, v)}=$ $\left\{u \in S: L^{\chi}(u) \geq C_{(\varpi, \delta, v)}(u)\right\}=\left\{i \in S: \alpha_{L}^{\chi}(u) \geq \lambda, \beta_{L}^{\chi} \geq \mu, \gamma_{L}^{\chi}(u) \leq \nu\right\}$ is called $a(\varpi, \delta, v)-$ level subset of $L^{\chi}$. 


\section{4. $\chi$-SINGLE VALUED NEUTROSOPHIC SUBGROUPS}

Definition 4..1. Consider $L^{\chi} \in \chi-S V N S G P(G)$ and assume $G$ be a group. Then $L^{\chi}$ is said to be $\chi$ single valued neutrosophic subgroup $(\chi-S V N S G)$ of $G$ if $L^{\chi}\left(u^{-1}\right) \geq L^{\chi}(u)$. i.e. $\alpha_{L}^{\chi}\left(u^{-1}\right) \geq \alpha_{L}^{\chi}(u)$, $\beta_{L}^{\chi}\left(u^{-1}\right) \geq \beta_{L}^{\chi}(u)$, and $\gamma_{L}^{\chi}\left(u^{-1}\right) \leq \gamma_{L}^{\chi}(u), \forall u \in G$.

Proposition 4..2. Let $\left\{L_{\eta}^{\chi}\right\}_{\eta \in \zeta} \subset \chi-S V N S G(G)$. Then $\bigcap_{\eta \in \zeta} L_{\eta}^{\chi} \in \chi-S V N S G(G)$.

Proposition 4..3. Let $L^{\chi}$ and $M^{\chi}$ be any two $\chi-S V N S G$ s of a group $G$. Then these are equivalent conditions: (1) $L^{\chi} \circ M^{\chi} \in \chi-S V N S G(G)$ (2) $L^{\chi} \circ M^{\chi}=M^{\chi} \circ L^{\chi}$.

Proof. Proof is obvious.

Proposition 4..4. Let $L^{\chi} \in \chi-S V N S G(G)$. Then $L^{\chi}\left(u^{-1}\right)=L^{\chi}(u)$, i.e. $\alpha_{L}^{\chi}\left(u^{-1}\right)=\alpha_{L}^{\chi}(u), \beta_{L}^{\chi}\left(u^{-1}\right)=$ $\beta_{L}^{\chi}(u), \gamma_{L}^{\chi}\left(u^{-1}\right)=\gamma_{L}^{\chi}(u)$ and $L(u) \leq L(e)$ i.e. $\alpha_{L}^{\chi}(u) \leq \alpha_{L}^{\chi}(e), \beta_{L}^{\chi}(u) \leq \beta_{L}^{\chi}(e), \gamma_{L}^{\chi}(u) \geq \gamma_{L}^{\chi}(e)$ for every $u \in G$, where e signify the identity element in $G$.

Proof. Suppose $u \in G$. So $\alpha_{L}^{\chi}(u)=\alpha_{L}^{\chi}\left(\left(u^{-1}\right)^{-1}\right) \geq \alpha_{L}^{\chi}\left(u^{-1}\right), \forall u \in G$. $\beta_{L}^{\chi}(u)=\beta_{L}^{\chi}\left(\left(u^{-1}\right)^{-1}\right) \geq \beta_{L}^{\chi}\left(u^{-1}\right)$ $, \forall u \in G \cdot \gamma_{L}^{\chi}(u)=\gamma_{L}^{\chi}\left(\left(u^{-1}\right)^{-1}\right) \leq \gamma_{L}^{\chi}\left(u^{-1}\right), \forall u \in G$. Since $L^{\chi} \in \chi-S V N S G(G), \alpha_{L}^{\chi}\left(u^{-1}\right) \geq \alpha_{L}^{\chi}(u)$, $\beta_{L}^{\chi}\left(u^{-1}\right) \geq \beta_{L}^{\chi}(u)$ and $\gamma_{L}^{\chi}\left(u^{-1}\right) \leq \gamma_{L}^{\chi}(u)$ for every $u \in G$. Hence $\alpha_{L}^{\chi}\left(u^{-1}\right)=\alpha_{L}^{\chi}(u), \beta_{L}^{\chi}\left(u^{-1}\right)=\beta_{L}^{\chi}(u)$, $\beta_{L}^{\chi}\left(u^{-1}\right)=\beta_{L}^{\chi}(u)$.(i.e.,) $L^{\chi}\left(u^{-1}\right)=L^{\chi}(u)$ Also, $\alpha_{L}^{\chi}(e)=\alpha_{L}^{\chi}\left(u u^{-1}\right) \geq \alpha_{L}^{\chi}(u) \wedge \alpha_{L}^{\chi}\left(u^{-1}\right)=\alpha_{L}^{\chi}(u)$, $\beta_{L}^{\chi}(e)=\beta_{L}^{\chi}\left(u u^{-1}\right) \geq \beta_{L}^{\chi}(u) \wedge \beta_{L}^{\chi}\left(u^{-1}\right)=\beta_{L}^{\chi}(u) \gamma_{L}^{\chi}(e)=\gamma_{L}^{\chi}\left(u u^{-1}\right) \leq \gamma_{L}^{\chi}(u) \wedge \gamma_{L}^{\chi}\left(u^{-1}\right)=\gamma_{L}^{\chi}(u)$ Hence $\alpha_{L}^{\chi}(u) \leq \alpha_{L}^{\chi}(e), \beta_{L}^{\chi}(u) \leq \beta_{L}^{\chi}(e), \gamma_{L}^{\chi}(u) \geq \gamma_{L}^{\chi}(e) \forall u \in G$. (i.e.,) $L^{\chi}(u) \leq L^{\chi}(e)$.

Proposition 4..5. If $L^{\chi} \in \chi-S V N S G(G)$, then $G_{L^{\chi}}=\left\{u \in G: L^{\chi}(u)=L^{\chi}(e)\right.$, i.e., $\alpha_{L}^{\chi}(u)=$ $\left.\alpha_{L}^{\chi}(e), \beta_{L}^{\chi}(u)=\beta_{L}^{\chi}(e), \gamma_{L}^{\chi}(u)=\gamma_{L}^{\chi}(e)\right\}$ is a subgroup of $G$.

Proof. Let $u, v \in G_{L \chi}$. Then $\alpha_{L}^{\chi}(u)=\alpha_{L}^{\chi}(e), \beta_{L}^{\chi}(u)=\beta_{L}^{\chi}(e), \gamma_{L}^{\chi}(u)=\gamma_{L}^{\chi}(e)$ and $\alpha_{L}^{\chi}(v)=\alpha_{L}^{\chi}(e), \beta_{L}^{\chi}(v)=$ $\beta_{L}^{\chi}(e), \gamma_{L}^{\chi}(v)=\gamma_{L}^{\chi}(e)$. Thus $\alpha_{L}^{\chi}\left(u v^{-1}\right) \geq \alpha_{L}^{\chi}(u) \wedge \alpha_{L}^{\chi}\left(v^{-1}\right)=\alpha_{L}^{\chi}(u) \wedge \alpha_{L}^{\chi}(v)$ by proposition $4 . .4=$ $\alpha_{L}^{\chi}(e) \wedge \alpha_{L}^{\chi}(e)=\alpha_{L}^{\chi}(e)$ Similarly $\beta_{L}^{\chi}\left(u v^{-1}\right) \geq \beta_{L}^{\chi}(e) \cdot \gamma_{L}^{\chi}\left(u v^{-1}\right) \leq \gamma_{L}^{\chi}(u) \vee \gamma_{L}^{\chi}\left(v^{-1}\right)=\gamma_{L}^{\chi}(u) \vee \gamma_{L}^{\chi}(v)$ by proposition $4 . .4=\gamma_{L}^{\chi}(e) \vee \gamma_{L}^{\chi}(e)=\gamma_{L}^{\chi}(e)$. Also, by proposition 4..4, $\alpha_{L}^{\chi}\left(u v^{-1}\right) \leq \alpha_{L}^{\chi}(e), \beta_{L}^{\chi}\left(u v^{-1}\right) \leq$ $\beta_{L}^{\chi}(e), \gamma_{L}^{\chi}\left(u v^{-1}\right) \geq \gamma_{L}^{\chi}(e)$. So, $\alpha_{L}^{\chi}\left(u v^{-1}\right)=\alpha_{L}^{\chi}(e), \beta_{L}^{\chi}\left(u v^{-1}\right)=\beta_{L}^{\chi}(e), \gamma_{L}^{\chi}\left(u v^{-1}\right)=\gamma_{L}^{\chi}(e)$. (i.e.,) $L^{\chi}\left(u v^{-1}\right)=L^{\chi}(e)$. Thus $u v^{-1} \in G_{L \chi}$. Hence $G_{L \chi}$ is a subgroup of $G$.

Proposition 4..6. Let $L^{\chi} \in \chi-S V N S G(G)$. If $L^{\chi}\left(u v^{-1}\right)=L^{\chi}(e)$.(i.e.,)

$\alpha_{L}^{\chi}\left(u v^{-1}\right)=\alpha_{L}^{\chi}(e), \beta_{L}^{\chi}\left(u v^{-1}\right)=\beta_{L}^{\chi}(e), \gamma_{L}^{\chi}\left(u v^{-1}\right)=\gamma_{L}^{\chi}(e)$ for any $u, v \in G$,

then $L^{\chi}(u)=L^{\chi}(v)$ (i.e.,) $\alpha_{L}^{\chi}(u)=\alpha_{L}^{\chi}(v), \beta_{L}^{\chi}(u)=\beta_{L}^{\chi}(v), \gamma_{L}^{\chi}(u)=\gamma_{L}^{\chi}(v)$

Proof. Let $u, v \in G_{L \chi}$. Then $\alpha_{L}^{\chi}(u)=\alpha_{L}^{\chi}\left(\left(u v^{-1}\right) v\right) \geq \alpha_{L}^{\chi}\left(u v^{-1}\right) \wedge \alpha_{L}^{\chi}(v)=\alpha_{L}^{\chi}(e) \wedge \alpha_{L}^{\chi}(v)=\alpha_{L}^{\chi}(v)$ Also, by proposition $4 . .4 \alpha_{L}^{\chi}\left(u^{-1}\right)=\alpha_{L}^{\chi}(u)$, then we have $\alpha_{L}^{\chi}\left(u v^{-1}\right)=\alpha_{L}^{\chi}\left(\left(v u^{-1}\right)^{-1}\right)=\alpha_{L}^{\chi}\left(v u^{-1}\right)$ and thus $\alpha_{L}^{\chi}(v)=\alpha_{L}^{\chi}\left(\left(v u^{-1}\right) u\right) \geq \alpha_{L}^{\chi}\left(v u^{-1}\right) \wedge \alpha_{L}^{\chi}(u)=\alpha_{L}^{\chi}\left(u v^{-1}\right) \wedge \alpha_{L}^{\chi}(u)=\alpha_{L}^{\chi}(e) \wedge \alpha_{L}^{\chi}(u)=\alpha_{L}^{\chi}(u)$. So $\alpha_{L}^{\chi}(u)=\alpha_{L}^{\chi}(v)$. Similarly, we have $\beta_{L}^{\chi}(u)=\beta_{L}^{\chi}(v), \gamma_{L}^{\chi}(u)=\gamma_{L}^{\chi}(v)$.

Proposition 4..7. $L^{\chi} \in \chi-S V N S G(G)$ if and only if $\alpha_{L}^{\chi}\left(u v^{-1}\right) \geq \alpha_{L}^{\chi}(u) \wedge \alpha_{L}^{\chi}(v), \beta_{L}^{\chi}\left(u v^{-1}\right) \geq \beta_{L}^{\chi}(u) \wedge \beta_{L}^{\chi}(v), \gamma_{L}^{\chi}\left(u v^{-1}\right) \leq \gamma_{L}^{\chi}(u) \vee \gamma_{L}^{\chi}(v)$ for any $u, v \in G$.

Proof. Using Definition $4 . .1$ and proposition $4 . .4$ we get the proof.

Proposition 4..8. The group G cannot be the union of two proper $\chi-S V N S G s$.

Proof. Let $L^{\chi}$ and $M^{\chi}$ are proper $\chi-S V N S G$ s of a group $G$ whenever $L^{\chi} \cup M^{\chi}=1_{N}, L^{\chi} \neq 1_{N}$ and $M^{\chi} \neq$ $1_{N} \cdot L^{\chi} \cup M^{\chi}=1_{N} \Rightarrow \alpha_{L}^{\chi} \vee \alpha_{M}^{\chi}=1, \beta_{L}^{\chi} \vee \beta_{M}^{\chi}=1, \gamma_{L}^{\chi} \wedge \gamma_{M}^{\chi}=0$. Then $\alpha_{L}^{\chi}=1$ or $\alpha_{M}^{\chi}=1, \beta_{L}^{\chi}=$ 1 or $\beta_{M}^{\chi}=1, \gamma_{L}^{\chi}=0$ or $\gamma_{M}^{\chi}=0$ Since $L^{\chi} \neq 1_{N}$ and $M^{\chi} \neq 1_{N}, \alpha_{L}^{\chi} \neq 1$ or $\beta_{L}^{\chi} \neq 1$ or $\gamma_{L}^{\chi} \neq 0$ and $\alpha_{M}^{\chi} \neq 1$ or $\beta_{M}^{\chi} \neq 1$ or $\gamma_{M}^{\chi} \neq 0$. In either cases, we get the contradiction.

Proposition 4..9. If $L^{\chi}$ is a $\chi-S V N S G P$ of a group $G$ then it is $\chi-S V N S G$ of $G$.

Proof. Suppose $u \in G$. Also $G$ has a order finite, Assume order of $u$ is $n$ (finite). $\Rightarrow u^{n}=e$, whereas $e$ indicate identity of $G$. Thus $u^{-1}=u^{n-1}$. Since $L^{\chi}$ is a $\chi-S V N S G P$ of a group $G$, Thus $\alpha_{L}^{\chi}\left(u^{-1}\right)=\alpha_{L}^{\chi}\left(u^{n-1}\right)=$ $\alpha_{L}^{\chi}\left(u^{n-2} u\right) \geq \alpha_{L}^{\chi}(u) \beta_{L}^{\chi}\left(u^{-1}\right)=\beta_{L}^{\chi}\left(u^{n-1}\right)=\beta_{L}^{\chi}\left(u^{n-2} u\right) \geq \beta_{L}^{\chi}(u), \gamma_{L}^{\chi}\left(u^{-1}\right)=\gamma_{L}^{\chi}\left(u^{n-1}\right)=\gamma_{L}^{\chi}\left(u^{n-2} i\right) \leq$ $\gamma_{L}^{\chi}(u)$. Hence $L^{\chi}$ is a $\chi-S V N S G$ of $G$. 
Proposition 4..10. Suppose $L^{\chi}$ be a $\chi-S V N S G$ of a group $G$ and let $u \in G$. Then $L^{\chi}(u v)=L^{\chi}(v)$ ,i.e. $\alpha_{L}^{\chi}(u v)=\alpha_{L}^{\chi}(u), \beta_{L}^{\chi}(u v)=\beta_{L}^{\chi}(u), \gamma_{L}^{\chi}(u v)=\gamma_{L}^{\chi}(u) \forall v \in G \Leftrightarrow L^{\chi}(u)=L^{\chi}(e)$. i.e. $\alpha_{L}^{\chi}(u)=$ $\alpha_{L}^{\chi}(e), \beta_{L}^{\chi}(u)=\beta_{L}^{\chi}(e), \gamma_{L}^{\chi}(u)=\gamma_{L}^{\chi}(e)$, where identity of $G$ is $e$.

Proof. Suppose $L^{\chi}(u v)=L^{\chi}(v)$ for every $v \in G$. Then obviously $L^{\chi}(u)=L^{\chi}(e)$. Conversely, considering $L^{\chi}(u)=L^{\chi}(e)$. Then by Proposition $4 . .4 \alpha_{L}^{\chi}(v) \leq \alpha_{L}^{\chi}(u), \beta_{L}^{\chi}(v) \leq \beta_{L}^{\chi}(u), \gamma_{L}^{\chi}(v) \leq \gamma_{L}^{\chi}(u) \forall v \in G$. Since $L^{\chi}$ is a $\chi-S V N S G$ of $G$, then $\alpha_{L}^{\chi}(u v) \geq \alpha_{L}^{\chi}(u) \wedge \alpha_{L}^{\chi}(v), \beta_{L}^{\chi}(u v) \geq \beta_{L}^{\chi}(u) \wedge \beta_{L}^{\chi}(v), \gamma_{L}^{\chi}(u v) \leq \gamma_{L}^{\chi}(u) \vee \gamma_{L}^{\chi}(v)$. Thus $\alpha_{L}^{\chi}(u v) \geq \alpha_{L}^{\chi}(v), \beta_{L}^{\chi}(u v) \geq \beta_{L}^{\chi}(v), \alpha_{L}^{\chi}(u v) \leq \gamma_{L}^{\chi}(v) \forall v \in G$. On the other hand, by Proposition $4 . .4$ $\alpha_{L}^{\chi}(v)=\alpha_{L}^{\chi}\left(u^{-1} u v\right) \geq \alpha_{L}^{\chi}(u) \wedge \alpha_{L}^{\chi}(u v), \beta_{L}^{\chi}(v) \geq \beta_{L}^{\chi}(u) \wedge \beta_{L}^{\chi}(u v), \gamma_{L}^{\chi}(v) \leq \gamma_{L}^{\chi}(u) \vee \gamma_{L}^{\chi}(u v)$. Since $\alpha_{L}^{\chi}(u) \geq \alpha_{L}^{\chi}(v), \beta_{L}^{\chi}(u) \geq \beta_{L}^{\chi}(v), \gamma_{L}^{\chi}(u) \leq \gamma_{L}^{\chi}(v) \forall v \in G \alpha_{L}^{\chi}(u) \wedge \alpha_{L}^{\chi}(u v)=\alpha_{L}^{\chi}(u v), \beta_{L}^{\chi}(u) \wedge \beta_{L}^{\chi}(u v)=$ $\beta_{L}^{\chi}(u v), \gamma_{L}^{\chi}(u) \vee \gamma_{L}^{\chi}(u v)=\gamma_{L}^{\chi}(u v)$. So $\alpha_{L}^{\chi}(v) \geq \alpha_{L}^{\chi}(u v), \beta_{L}^{\chi}(v) \geq \beta_{L}^{\chi}(u v), \gamma_{L}^{\chi}(v) \leq \alpha_{L}^{\chi}(u v) \forall v \in G$. Hence $\alpha_{L}^{\chi}(u v)=\alpha_{L}^{\chi}(v), \beta_{L}^{\chi}(u v)=\beta_{L}^{\chi}(v), \gamma_{L}^{\chi}(u v)=\gamma_{L}^{\chi}(v), \forall v \in G$.

Proposition 4..11. Let define a group homomorphism $g: G \rightarrow G^{\prime}$, whereas $L^{\chi} \in \chi-S V N S G(G), M^{\chi} \in \chi-$ $S V N S G\left(G^{\prime}\right)$. Then these conditions must be satisfy:

(i) $L^{\chi}$ contain the sup-property $\Rightarrow g\left(L^{\chi}\right) \in \chi-S V N G\left(G^{\prime}\right)$.

(ii) $g^{-1}\left(M^{\chi}\right) \in \chi-S V N S G(G)$.

Proof. (i) By Proposition, Assume groupoid homomorphism $g: G \rightarrow G^{\prime \prime}$ also consider $L^{\chi} \in \chi-S V N S(G)$ contain the sup property. (1) $L^{\chi} \in \chi-S V N S G P(G) \Rightarrow g\left(L^{\chi}\right) \in \chi-S V N S G P\left(G^{\prime \prime}\right)$. (2) If $L^{\chi}$ is a $\chi-$ $S V N I(\chi-S V N L I, \chi-S V N R I)$ of $G$, then $g\left(L^{\chi}\right)$ is a $\chi-S V N I(\chi-S V N L I, \chi-S V N R I)$ of $G^{\prime \prime}$. Since $g\left(L^{\chi}\right) \in \chi-S V N S G P(G)$, it is enough indicate that $\alpha_{g(L)}\left(v^{-1}\right) \geq \alpha_{g(L)}(v), \beta_{g(L)}\left(v^{-1}\right) \geq \beta_{g(L)}(v), \gamma_{g(L)}$ $\left(v^{-1}\right) \leq \gamma_{g(L)}(v), \forall v \in g(G)$. Let $v \in g(G)$. Then $\phi \neq g^{-1}(v) \subset G$. Since $L^{\chi}$ has the sup-property, $\exists$ $u_{0} \in g^{-1}(v)$ for that

$$
\begin{aligned}
& \alpha_{L}^{\chi}\left(u_{0}\right)=\bigvee_{t \in g^{-1}(v)} \alpha_{L}^{\chi}(t), \quad \beta_{L}^{\chi}\left(u_{0}\right)=\bigvee_{t \in g^{-1}(v)} \beta_{L}^{\chi}(t), \gamma_{L}^{\chi}\left(u_{0}\right)=\bigwedge_{t \in g^{-1}(v)} \gamma_{L}^{\chi}(t), \\
& \alpha_{g(L)}\left(v^{-1}\right)=g\left(\alpha_{L}^{\chi}\right)\left(v^{-1}\right)=\bigvee_{t \in g^{-1}\left(v^{-1}\right)} \alpha_{L}^{\chi}(t) \geq \alpha_{L}^{\chi}\left(u_{0}^{-1}\right) \geq \alpha_{L}^{\chi}\left(u_{0}\right), \alpha_{g(L)}(v) . \\
& \beta_{g(L)}\left(v^{-1}\right)=g\left(\beta_{L}^{\chi}\right)\left(v^{-1}\right)=\bigvee_{t \in g^{-1}\left(v^{-1}\right)} \beta_{L}^{\chi}(t) \geq \beta_{L}^{\chi}\left(u_{0}^{-1}\right) \geq \beta_{L}^{\chi}\left(u_{0}\right), \beta_{g(L)}(v) . \\
& \gamma_{g(L)}\left(v^{-1}\right)=g\left(\gamma_{L}^{\chi}\right)\left(v^{-1}\right)=\bigwedge_{t \in g^{-1}\left(v^{-1}\right)} \gamma_{L}^{\chi}(t) \leq \gamma_{L}^{\chi}\left(u_{0}^{-1}\right) \leq \gamma_{L}^{\chi}\left(u_{0}\right), \gamma_{g(L)}(v) .
\end{aligned}
$$

Hence $g\left(L^{\chi}\right) \in \chi$-SVNSG(G). (ii) By Proposition in [18], we have a groupoid homomorphism $g: G \rightarrow G^{\prime \prime}$ and suppose $M^{\chi} \in \chi-S V N S\left(G^{\prime \prime}\right)$ (1) If $M^{\chi} \in \chi-S V N S G P\left(G^{\prime \prime}\right)$, then $g^{-1}\left(M^{\chi}\right) \in \chi$ $S V N S G P(G)$. (2) If $M^{\chi}$ is a $\chi-S V N I(\chi-S V N L I, \chi-S V N R I)$ of $G^{\prime \prime}$ then $g^{-1}\left(M^{\chi}\right)$ is a $\chi-S V N I(\chi-$ $S V N L I, \chi-S V N R I)$ of $G$. Since $g^{-1}\left(M^{\chi}\right) \in \chi-S V N S G P(G)$, It is adequate to express $g^{-1}\left(M^{\chi}\right)\left(u^{-1}\right) \geq$ $g^{-1}\left(M^{\chi}\right)(u) \forall u \in G$. Let $u \in G$. Then $\alpha_{g^{-1}(M)}^{\chi}\left(u^{-1}\right)=g^{-1}\left(\alpha_{M}^{\chi}\right)\left(u^{-1}\right)=\alpha_{M}^{\chi}\left(g\left(\left(u^{-1}\right)\right)=\alpha_{M}^{\chi}\left(\left((g(u))^{-1}\right)\right.\right.$ $\geq \alpha_{M}^{\chi}(g(u))=\alpha_{g^{-1}(M)}(u), \beta_{g^{-1}(M)}\left(u^{-1}\right)=g^{-1}\left(\beta_{M}^{\chi}\right)\left(u^{-1}\right)=\beta_{M}^{\chi}\left(g\left(\left(u^{-1}\right)\right)=\beta_{M}^{\chi}\left(\left((g(u))^{-1}\right) \geq\right.\right.$ $\beta_{M}^{\chi}(g(u))=\beta_{g^{-1}(M)}(u), \gamma_{g^{-1}(M)}\left(u^{-1}\right)=g^{-1}\left(\gamma_{M}^{\chi}\right)\left(u^{-1}\right)=\gamma_{M}^{\chi}\left(g\left(\left(u^{-1}\right)\right)=\gamma_{M}^{\chi}\left(\left((g(u))^{-1}\right) \leq \gamma_{M}^{\chi}(g(u))\right.\right.$ $=\gamma_{g^{-1}(M)}(u)$. Hence $g^{-1}\left(M^{\chi}\right) \in \chi-S V N S G(G)$.

Proposition 4..12. Let $L^{\chi}$ be a $\chi-S V N S G$ of a group $G$. Then for every $(\varpi, \delta, v) \in \beta^{3}$ with $(\varpi, \delta, v) \leq$ $L^{\chi}(e)$, (i.e.,) $\varpi \leq \alpha_{L}^{\chi}(e), \delta \leq \beta_{L}^{\chi}(e), v \geq \gamma_{L}^{\chi}(e), G_{L \chi}^{(\varpi, \delta, v)}$ is a subgroup of $G$, where e represent the identity of $G$.

Proof. Clearly, $G_{L^{\chi}}^{(\varpi, \delta, v)} \neq \phi$. Let $u, v \in G_{L^{\chi}}^{(\varpi, \delta, v)}$. Then $L^{\chi}(u) \geq(\varpi, \delta, v)$ and $L^{\chi}(v) \geq(\varpi, \delta, v)$. (i.e.,) $\alpha_{L}^{\chi}(u) \geq \varpi, \beta_{L}^{\chi}(u) \geq \delta, \gamma_{L}^{\chi}(u) \leq v$ and $\alpha_{L}^{\chi}(v) \geq \varpi, \beta_{L}^{\chi}(v) \geq \delta, \gamma_{L}^{\chi}(v) \leq v$. Since $L^{\chi} \in \chi-S V N S G(G)$, $\alpha_{L}^{\chi}(u v) \geq \alpha_{L}^{\chi}(u) \wedge \alpha_{L}^{\chi}(v) \geq \varpi, \beta_{L}^{\chi}(u v) \geq \beta_{L}^{\chi}(u) \wedge \beta_{L}^{\chi}(v) \geq \delta, \gamma_{L}^{\chi}(u v) \leq \gamma_{L}^{\chi}(u) \vee \gamma_{L}^{\chi}(v) \leq v$. Thus $L^{\chi}(u v) \geq(\varpi, \delta, v)$. So $u v \in G_{L^{\chi}}^{(\varpi, \delta, v)}$. On the other hand, $\alpha_{L}^{\chi}\left(u^{-1}\right) \geq \alpha_{L}^{\chi}(u) \geq \varpi, \beta_{L}^{\chi}\left(u^{-1}\right) \geq \beta_{L}^{\chi}(u) \geq$ $\delta, \gamma_{L}^{\chi}\left(u^{-1}\right) \leq \gamma_{L}^{\chi}(u) \leq v$. Thus $L^{\chi}\left(u^{-1}\right) \geq(\varpi, \delta, v)$. So $u^{-1} \in G_{L^{\chi}}^{(\varpi, \delta, v)}$. Hence $G_{L^{\chi}}^{(\varpi, \delta, v)}$ is a subgroup of G.

Proposition 4..13. Assume $L^{\chi}$ be a $\chi-S V N S$ in a group $G$ such that $G_{L^{\chi}}^{(\varpi, \delta, v)}$ is a subgroup of $G$ for each $(\varpi, \delta, v) \in \beta^{3}$ with $(\varpi, \delta, v) \leq L^{\chi}(e)$. Then $L^{\chi}$ is a $\chi$-SVNSG of a group $G$.

Proof. For any $u, v \in G$, let $L^{\chi}(u)=\left(t_{1}, s_{1}, r_{1}\right)$ and let $L^{\chi}(v)=\left(t_{2}, s_{2}, r_{2}\right)$. Then clearly, $u \in G_{L^{\chi}}^{\left(t_{1}, s_{1}, r_{1}\right)}$ and $v \in G_{L^{\chi}}^{\left(t_{2}, s_{2}, r_{2}\right)}$. Suppose $t_{1}<t_{2}, s_{1}<s_{2}$ and $r_{1}>r_{2}$. Then $G_{L^{\chi}}^{\left(t_{2}, s_{2}, r_{2}\right)} \subset G_{L^{\chi}}^{\left(t_{1}, s_{1}, r_{1}\right)}$. Thus $v \in G_{L^{\chi}}^{\left(t_{1}, s_{1}, r_{1}\right)}$. Since $G_{L^{\chi}}^{\left(t_{1}, s_{1}, r_{1}\right)}$ is a subgroup of $G, u v \in G_{L^{\chi}}^{\left(t_{1}, s_{1}, r_{1}\right)}$. Then $L^{\chi}(u v)=\left(t_{1}, s_{1}, r_{1}\right)$. (i.e.,) 
$\alpha_{L}^{\chi}(u v) \geq t_{1}, \beta_{L}^{\chi}(u v) \geq s_{1}, \gamma_{L}^{\chi}(u v) \leq r_{1}$. So $\alpha_{L}^{\chi}(u v) \geq \alpha_{L}^{\chi}(u) \wedge \alpha_{L}^{\chi}(v), \beta_{L}^{\chi}(u v) \geq \beta_{L}^{\chi}(u) \wedge \beta_{L}^{\chi}(v), \gamma_{L}^{\chi}(u v) \leq$ $\gamma_{L}^{\chi}(u) \vee \gamma_{L}^{\chi}(v)$ For each $u \in G$. let $L^{\chi}(u v)=(\varpi, \delta, v)$. Then $u \in G_{L^{\chi}}^{(\varpi, \delta, v)}$. Since $G_{L^{\chi}}^{(\varpi, \delta, v)}$ is a subgroup of $G, u^{-1} \in G_{L^{\chi}}^{(\varpi, \delta, v)}$. So $L^{\chi}\left(u^{-1}\right) \geq(\varpi, \delta, v)$. (i.e.,) $\alpha_{L}^{\chi}\left(u^{1}\right) \geq \alpha_{L}^{\chi}(u), \beta_{L}^{\chi}\left(u^{1}\right) \geq \beta_{L}^{\chi}(u), \gamma_{L}^{\chi}\left(u^{1}\right) \leq \gamma_{L}^{\chi}(u)$. Hence $L^{\chi}$ is a $\chi-S V N S G$ of a group $G$.

Proposition 4..14. Let $L^{\chi}$ be a $\chi$-SVNS in $S$, suppose $\left(\varpi_{1}, \delta_{1}, v_{1}\right),\left(\varpi_{2}, \delta_{2}, v_{2}\right) \in \operatorname{Im}\left(L^{\chi}\right)$. If $\varpi_{1}<$ $\varpi_{2}, \delta_{1}<\delta_{2}, v_{1}<v_{2}$ then $L^{\chi\left(\varpi_{1}, \delta_{1}, v_{1}\right)} \supset L^{\chi\left(\varpi_{2}, \delta_{2}, v_{2}\right)}$.

Proposition 4..15. Let $L^{\chi}$ be a $\chi-S V N S$ in a group $G$.Then $L^{\chi}$ is a $\chi-S V N S G$ of $G \Leftrightarrow L^{\chi(\varpi, \delta, v)}$ is a subgroup of $G$ for every $(\varpi, \delta, v) \in \operatorname{Im}\left(L^{\chi}\right)$.

Definition 4..16. Let $L^{\chi}$ be a $\chi-S V N S G$ of group $G$ and consider $(\varpi, \delta, v) \in \operatorname{Im}\left(L^{\chi}\right)$. Then subgroup $L^{\chi(\varpi, \delta, v)}$ is known a $(\varpi, \delta, v)$-level subgroup of $L^{\chi}$.

Lemma 4..17. Assume $L^{\chi}$ be any $\chi-S V N S$ in $S$.

Then $\alpha_{L}^{\chi}(u)=\bigvee\left\{\varpi: u \in L^{\chi(\varpi, \delta, v)}\right\}, \beta_{L}^{\chi}(u)=\bigvee\left\{\delta: u \in L^{\chi(\varpi, \delta, v)}\right\}, \gamma_{L}^{\chi}(u)=\bigwedge\left\{v: u \in L^{\chi(\varpi, \delta, v)}\right\}$. where $u \in S$ and $(\varpi, \delta, v) \in \beta^{3}$ with $\varpi+\delta+v \leq 3$.

Proof. Let $\pi=\bigvee\left\{\varpi: u \in L^{\chi(\varpi, \delta, v)}\right\}, \psi=\bigvee\left\{\delta: u \in L^{\chi(\varpi, \delta, v)}\right\}, \theta=\wedge\left\{v: u \in L^{\chi(\varpi, \delta, v)}\right\}$ and let $\epsilon>0$ be arbitrary. Then $\pi-\epsilon<\bigvee\left\{\varpi: u \in L^{\chi(\varpi, \delta, v)}\right\}, \psi-\epsilon<\bigvee\left\{\delta: u \in L^{\chi(\varpi, \delta, v)}\right\}, \theta+\epsilon>$ $\wedge\left\{\varpi: u \in L^{\chi(\varpi, \delta, v)}\right\}$. Thus $\exists(\varpi, \delta, v) \in \psi$ with $\varpi+\delta+v \leq 3$ such that $u \in L^{\chi(\varpi, \delta, v)}, \pi-\epsilon<$ $\varpi, \psi-\epsilon<\delta, \theta+\epsilon>v$. Since $u \in L^{\chi(\varpi, \delta, v)}, \pi_{L}^{\chi}(u) \geq \varpi, \psi_{L}^{\chi}(u) \geq \delta, \theta_{L}^{\chi}(u) \leq v$. Thus $\pi_{L}^{\chi}(u) \geq$ $\pi-\epsilon, \psi_{L}^{\chi}(u) \geq \psi-\epsilon, \theta_{L}^{\chi}(u) \leq \theta-\epsilon$. Since $\epsilon>0$ is arbitrary $, \pi_{L}^{\chi}(u) \geq \pi, \psi_{L}^{\chi}(u) \geq \psi, \theta_{L}^{\chi}(u) \leq \theta$ We show that $\pi_{L}^{\chi}(u) \leq \pi, \psi_{L}^{\chi}(u) \leq \psi, \theta_{L}^{\chi}(u) \geq \theta$. Let $\pi_{L}^{\chi}(u)=t_{1}, \psi_{L}^{\chi}(u)=t_{2}, \theta_{L}^{\chi}(u)=t_{3}$. Then $t_{1}+t_{2}+t_{3} \leq 3$. Thus $u \in L^{\chi\left(t_{1}, t_{2}, t_{3}\right)}$. So $t_{1} \in\left\{\varpi: u \in L^{\chi(\varpi, \delta, v)}\right\}, t_{2} \in\left\{\delta: u \in L^{\chi(\varpi, \delta, v)}\right\}, t_{3} \in\left\{v: u \in L^{\chi(\varpi, \delta, v)}\right\}$. Thus $t_{1} \leq \bigvee\left\{\varpi: u \in L^{\chi(\varpi, \delta, v)}\right\}, t_{2} \leq \bigvee\left\{\delta: u \in L^{\chi(\varpi, \delta, v)}\right\}, t_{3} \geq \bigwedge\left\{v: u \in L^{\chi(\varpi, \delta, v)}\right\}$. (i.e.,) $\pi_{L}^{\chi}(u) \leq \pi, \psi_{L}^{\chi}(u) \leq \psi, \theta_{L}^{\chi}(u) \geq \theta$. We should indicate by $\left(L^{\chi}\right)$ the $\chi-S V N S G$ generated by the fuzzy $\chi$-neutrosophic set $L^{\chi}$ in $G$. Similarly $\left(L^{\chi(\varpi, \delta, v)}\right)$ for the level subset $L^{\chi(\varpi, \delta, v)}$.

Lemma 4..18. Let $G$ be a group of order finite. Suppose $\exists$ is a $\chi-S V N S G L^{\chi}$ of $G$ that meets these conditions: for any $u, v \in G$, (i) $L^{\chi}(u)=L^{\chi}(v) \Rightarrow(u)=(v)$.

(ii) $\pi_{L}^{\chi}(u)>\pi_{L}^{\chi}(v), \psi_{L}^{\chi}(u)>\psi_{L}^{\chi}(v), \theta_{L}^{\chi}(u)<\theta_{L}^{\chi}(v) \Rightarrow(u) \subset(v)$. Then $G$ is a cyclic.

Proof. Consider $L^{\chi}$ is constant on $G$. So $L^{\chi}(u)=L^{\chi}(v) \Rightarrow(u)=(v)$. By (u), $(u)=(v)$. $\Rightarrow G=(u)$. Assume $L^{\chi}$ is not constant on $G$. Assume $\operatorname{Im}\left(L^{\chi}\right)=\left\{\left(t_{0}, s_{0}, r_{0}\right),\left(t_{1}, s_{1}, r_{1}\right), \ldots,\left(t_{n}, s_{n}, r_{n}\right)\right\}$, where $t_{0}>$ $t_{1}>\ldots>t_{n}, s_{0}>s_{1}>\ldots>s_{n}, r_{0}<r_{1}<\ldots<r_{n}$. Using proposition 4..14, 4..15, we attain the chain of

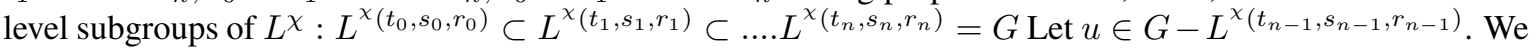
have to show $G=(u)$. Let $g \in G-L^{\chi}\left(t_{n-1}, s_{n-1}, r_{n-1}\right)$. Since $t_{0}>t_{1}>\ldots>t_{n}, s_{0}>s_{1}>\ldots>s_{n}, t_{0}<$ $t_{1}<\ldots<t_{n}, L^{\chi}(g)=L^{\chi}(u)=L^{\chi}\left(t_{n-1}, s_{n-1}, r_{n-1}\right)$. By $(\mathrm{u}),(g)=(u)$. Thus $G-L^{\chi}\left(t_{n-1}, s_{n-1}, r_{n-1}\right) \subset$ $(u)$. Now assume $g \in L^{\chi}\left(t_{n-1}, s_{n-1}, r_{n-1}\right)$. Then $\pi_{L}^{\chi}(g) \geq t_{n-1}>t_{n}=\pi_{L}^{\chi}(u), \psi_{L}^{\chi}(g) \geq s_{n-1}>s_{n}=$ $\psi_{L}^{\chi}(u), \theta_{L}^{\chi}(g) \leq r_{n-1}<r_{n}=\theta_{L}^{\chi}(u)$. By (ii), $(g) \subset(u)$. Thus $L^{\chi}\left(t_{n-1}, s_{n-1}, r_{n-1}\right) \subset(u)$. So $G=(u)$. So in each case, $G$ is cyclic.

Lemma 4..19. Suppose $p^{n}$ be the order of group $G$ such that $p$ is prime. Then $\exists$ a $\chi$-SVNSG $L^{\chi}$ of $G$ Complying with the following conditions: every $u, v \in G$,

(i) $L^{\chi}(u)=L^{\chi}(v) \Rightarrow(u)=(v)$

(ii) $\pi_{L}^{\chi}(u)>\pi_{L}^{\chi}(v), \psi_{L}^{\chi}(u)>\psi_{L}^{\chi}(v), \theta_{L}^{\chi}(u)<\theta_{L}^{\chi}(v) \Rightarrow(u) \subset(v)$.

Proof. Assume chain of following subgroup of $G:(e)=G_{0} \subset G_{1} \subset \ldots \subset G_{n-1} \subset G_{n}=G$, where $G_{u}$; the collection of subgroup of $G$ and generated by element with order $p^{u}, i=0,1, \ldots n$ whereas $e$ is the identity of $G$. We construct a complex mapping $L^{\chi}=\left(\pi_{L}, \psi_{L}, \theta_{L}\right): G \rightarrow \psi^{3}$ as: for every $u \in G$, $L^{\chi}(e)=\left(t_{0}, s_{0}, r_{0}\right)$ and $L^{\chi}(u)=\left(t_{u}, s_{u}, r_{u}\right)$ if $u \in G_{u}-G_{i-1}$ for any $i=1,2, \ldots n$, where $t_{u}, s_{u}, r_{u} \in \chi$ such that $t_{u}+s_{u}+r_{u} \leq 3, t_{0}>t_{1}>\ldots>t_{n}, s_{0}>s_{1}>\ldots>s_{n}, r_{0}<r_{1}<\ldots<r_{n}$. We can then easily verify that $L^{\chi}$ is a $\chi-S V N S G$ of $G$ sustaining both conditions. From Lemma $4 . .18$ and Lemma 4..19, get necessary result.

\section{CONCLUSION}

In this article, we give the notion of $\chi-S V N S s$ and subgroups. We investigate several operations and algebraic properties related to these ideas. In future work, researchers may extend this idea in topological spaces, rings, ideals, fields, and vector spaces. 


\section{REFERENCES}

[1] L. A. Zadeh, "Fuzzy sets," Information and control, vol. 8, no. 3, pp. 338-353, 1965, doi: 10.1016/S00199958(65)90241-X

[2] K. T. Atanassov, "Intuitionistic fuzzy sets," In Studies in Fuzziness and Soft Computing, Physica, Heidelberg, pp. 1-137, 1999, doi: 10.1007/978-3-7908-1870-3_1.

[3] D. Molodtsov, "Soft set theory-first results," Computers \& Mathematics with Applications, vol. 37, no. 4-5, pp. 19-31, 1999, doi: 10.1016/S0898-1221(99)00056-5.

[4] M. I. Ali, F. Feng, X. Liu, W. K. Min, and M. Shabir, "On some new operations in soft set theory," Computers \& Mathematics with Applications, vol. 57, no. 9, pp. 1547-1553, 2009, doi: 10.1016/j.camwa.2008.11.009.

[5] R. R. Yager, "Pythagorean fuzzy subsets," In 2013 Joint IFSA World Congress and NAFIPS Annual Meeting (IFSA/NAFIPS), 2013, pp. 57-61, doi: 10.1109/IFSA-NAFIPS.2013.6608375.

[6] R. R. Yager and A. M. Abbasov, "Pythagorean membership grades, complex numbers, and decision making," International Journal of Intelligent Systems, vol. 28, no. 5, pp. 436-452, 2013, doi: 10.1002/int.21584.

[7] R. R. Yager, "Pythagorean Membership Grades in Multicriteria Decision Making," in IEEE Transactions on Fuzzy Systems, vol. 22, no. 4, pp. 958-965, Aug. 2014, doi: 10.1109/TFUZZ.2013.2278989.

[8] X. Peng, H. Yuan, and Y. Yang, "Pythagorean fuzzy information measures and their applications," International Journal of Intelligent Systems, vol. 32, no. 10, pp. 991-1029, 2017, doi: 10.1002/int.21880.

[9] X. D. Peng, Y. Yang, J. Song, and Y. Jiang, "Pythagorean fuzzy soft set and its application," Computer Engineering, vol. 41, no. 7, pp. 24-229, 2015.

[10] A. M. Khalil, "Commentary on Generalized intuitionistic fuzzy soft sets with applications in decision-making," Applied Soft Computing, vol. 37, pp. 519-520, 2015, doi: 10.1016/j.asoc.2015.08.054.

[11] S. Alkhazaleh, A. R. Salleh, and N. Hassan, "Possibility fuzzy soft set," Advances in Decision Sciences, vol. 2011, 2011, doi: 10.1155/2011/479756.

[12] H. Zhang, L. Xiong, and W. Ma, “On interval-valued hesitant fuzzy soft sets,” Mathematical Problems in Engineering, vol. 2015, 2015, doi: 10.1155/2015/254764.

[13] H. D. Zhang and L. Shu, "Dual hesitant fuzzy soft set and its properties," In Fuzzy Systems \& Operations Research and Management, vol. 367, pp. 171-182, 2016, doi: 10.1007/978-3-319-19105-8_17.

[14] S. Sebastian and T. V. Ramakrishnan, "Multi-fuzzy sets: An extension of fuzzy sets," Fuzzy Information and Engineering, vol. 3, no. 1, pp. 35-43, 2011, doi: 10.1007/s12543-011-0064-y.

[15] I. Arockiarani and J. M. Jency, "More on fuzzy neutrosophic sets and fuzzy neutrosophic topological spaces," International journal of innovative research and studies, vol. 3, no. 5, pp. 643-652, 2014.

[16] I. Arockiarani, J. M. Jency, and F. N. On, "Continuity in Fuzzy Neutrosophic Topological spaces," Asian academic research journal of multidisciplinary, vol. 1, no. 24, pp. 330-340, 2014.

[17] I. Arockiarani and I. R. Sumathi, "Fuzzy neutrosophic groups," Advances in Fuzzy mathematics, vol. 10, no. 2, pp. 117-122, 2015.

[18] J. M. Jency and I. Arockiarani, “Fuzzy Neutrosophic Subgroups," Infinite Study, pp. 1-12, 2016.

[19] B. Banerjee, "Intuitionistic fuzzy subrings and ideals," J. Fuzzy Math., vol. 11, no. 1, pp. 139-155, 2003.

[20] K. Hur, S. Y. Jang, and H. W. Kang, "Intuitionistic fuzzy subgroupoids," International Journal of Fuzzy Logic and Intelligent Systems, vol. 3, no. 1, pp. 72-77, 2003, doi: 10.5391/IJFIS.2003.3.1.072.

[21] K. Hur, H. W. Kang, and H. K. Song, "Intuitionistic fuzzy subgroups and subrings," Honam mathematical journal, vol. 25, no. 1, pp. 19-41, 2003.

[22] K. Hur, S. Y. Jang, and H. W. Kang, "Intuitionistic fuzzy subgroups and cosets," Honam mathematical journal, vol. 26, no. 1, pp. 17-41, 2004.

[23] A. Rosenfeld, "Fuzzy groups," Journal of mathematical analysis and applications, vol. 35, no. 3, pp. 512-517, 1971.

[24] F. Smarandache, "Neutrosophic set-a generalization of the intuitionistic fuzzy set," International journal of pure and applied mathematics, vol. 24, no. 3, pp. 287-297, 2005.

[25] L. A. Zadeh, "Fuzzy sets," In Fuzzy sets, fuzzy logic, and fuzzy systems, vol. 6, pp. 394-432, 1996, doi: 10.1142/9789814261302_0021.

[26] F. Smarandache, “A unifying field in Logics: Neutrosophic Logic," In Philosophy: American Research Press, pp. $1-141,1999$.

[27] H. Wang, P. Madiraju, Y. Zhang, and R. Sunderraman, "Interval neutrosophic sets," General Mathematics, 2004, arXiv: math/0409113v1.

[28] S. Broumi, F. Smarandache, and M. Dhar, "Rough neutrosophic sets," Infinite Study, 2014.

[29] M. S. Hameed, S. Mukhtar, H. N. Khan, S. Ali, M. H. Mateen, and M. Gulzar, "Pythagorean Fuzzy N-Soft Groups," International Journal of Electrical and Computer Engineering (IJECE), vol. 21, no. 2, pp. 1030-1038, 2021, doi: 10.11591/ijeecs.v21.i2.pp1030-1038. 